\title{
Structural and biosynthetic analysis of the fabrubactins, unusual siderophores from Agrobacterium fabrum strain C58
}

\author{
Vladimir Vinnik ${ }^{1,5}$, Fan Zhang ${ }^{2}$, Hyunjun Park ${ }^{1,3}$, Taylor B. Cook ${ }^{4}$, Kurt Throckmorton ${ }^{1}$, \\ Brian F. Pfleger ${ }^{4}$, Tim S. Bugni², Michael G. Thomas ${ }^{1 *}$
}

${ }^{1}$ Department of Bacteriology, University of Wisconsin-Madison, Madison, Wisconsin 53706, USA

2Pharmaceutical Sciences Division, University of Wisconsin-Madison, Madison, Wisconsin 53705, USA

${ }^{3}$ Current address: CATALOG, Boston, Massachusetts 02129, USA

${ }^{4}$ Department of Chemical and Biological Engineering, University of Wisconsin-Madison, Madison, WI 53706, USA

${ }^{5}$ Current address: Department of Chemistry, University of Utah, Salt Lake City, Utah, 84112

*Correspondence: michael.thomas@wisc.edu (M.G.T) 


\section{TABLE OF CONTENTS:}

\section{Experimental Procedures:}

Plasmid and Bacterial Strain Construction........................................................ 3

Overproduction and purification of NPRS and PKS components............................. pg. 3

ATP-[32P]PP $P_{\mathrm{i}}$ exchange assays to determine $A$ domain specificity ......................... pg. 4

ATP-[32P]PP $P_{i}$ exchange assays to determine A domain specificity of FbnD-S- $\beta$-Ala..........pg. 4

Radioactive assays of $\mathrm{FbnF}$ and $\mathrm{FbnN}$ with malonyl-CoA or

$(2 R, S)$-methylmalonyl-CoA pg. 4

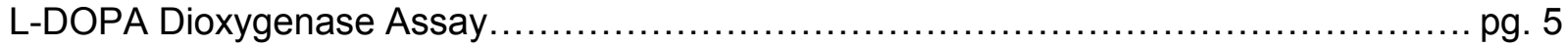

In vitro L-DOPA formation assays................................................................ 6

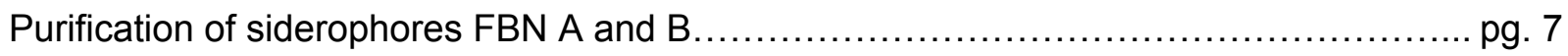

General experimental procedures for structure elucidation................................. pg. 8

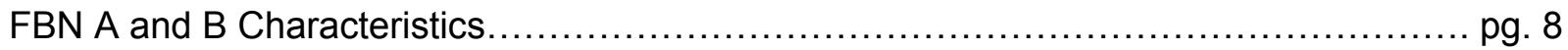

\section{Figures:}

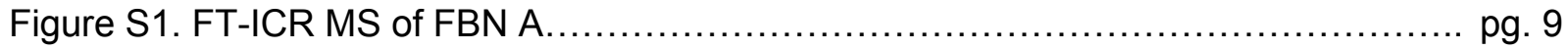

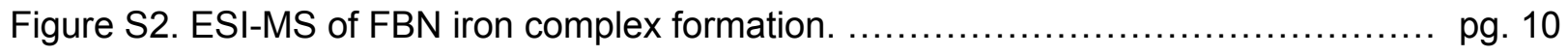

Figure S3. Characteristic fragments in the ESI-MS ${ }^{2}$ spectrum of FBN A and FBN B...... pg. 11

Figure S4. Substrate specificity of NRPS A domains and Acyl-CoA ligase domain.......... pg. 12

Figure S5. Evaluating FbnD activation of $\beta$-Ala and L-Ala after FbnD-S- $\beta$-Ala formation. pg. 13

Figure S6. Substrate specificities of the AT domains of FbnF and FbnN .................. pg. 14

Figure S7. Pigment production by overproduced FbnL/M in E. coli........................ pg. 15

Tables:

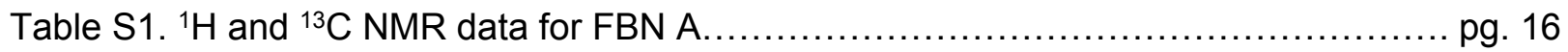

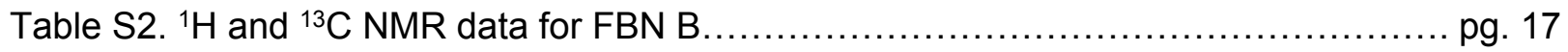

Table S3. Predicted functions of ORFs in the $f b n$ gene cluster.............................. pg. 18

Table S4. Kinetic parameters of FbnO-1 for activation of select amino acid substrates.... pg. 19

Table S5. Proposed Dmaq-containing siderophore BGC locus tags shown in Figure 5..... pg. 20

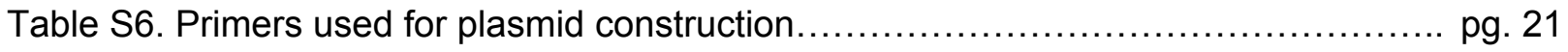

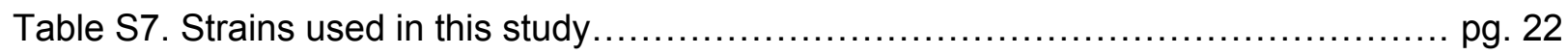

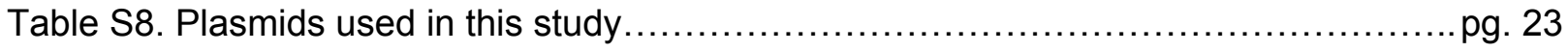




\section{EXPERIMENTAL METHODS}

Plasmid and bacterial strain construction. The NRPS, PKS, and acyl-CoA ligase domains were cloned into the overexpression vector pET28b (EMD chemicals) and the MLP was cloned into the vector pACYCDuet-1 (Novagen) using standard PCR-based methods as previously described ${ }^{1}$. The primers used for PCR amplification (Table S6) were designed so that overexpression from $\mathrm{pET} 28 \mathrm{~b}$ produced a protein with a hexahistidine tag at the $\mathrm{N}$-terminus except for FbnP which had a C-terminal tag. The strain $A$. fabrum C58 $\Delta f b n N$ was constructed with a pK18mobsacB plasmid using a sucrose counter-selection. Complementation of the $\Delta f b n N$ strain was done with pBBR1MCS-2$f b n N^{2,3}$.

Overproduction and purification of NPRS and PKS components. All proteins were overproduced in Escherichia coli BL21(DE3)ybdZ::aac(3)-IV and purified using nickel chelate chromatography, as previously described ${ }^{1}, 4$. We co-produced the NRPS components with the MLP FbnK. Some constructs were extended to include other domains flanking the $\mathrm{A}$ domains: the $\mathrm{A}-\mathrm{T}$ pair of $\mathrm{FbnD}$, the entire protein $\mathrm{FbnH}$, the condensation (C)-A1-T1 domains of FbnO module one, the cyclization (Cy)-A2-oxidation (Ox)-T2 domains of FbnO module two, the full protein $\mathrm{FbnP}$, and the C-A2-T2 of FbnP module two. The PKSs, encoded by $f b n F$ and $f b n N$, were overproduced as an entire module. 
ATP-[ $\left.{ }^{32} \mathrm{P}\right] \mathrm{PP}_{\mathrm{i}}$ exchange assays to determine $\mathrm{A}$ domain specificity. Amino aciddependent ATP-[32P]PP ${ }_{\mathbf{i}}$ exchange assays were done as previously described ${ }^{1,4}$. The purified proteins $\mathrm{FbnO}$ and $\mathrm{FbnH}$ were assayed for ability to active the 20 proteinogenic amino acids and FbnH was also assayed with L-DOPA. FbnD was assayed with Gly, DAla, L-Ala, $\beta$-Ala, and $\beta$-Ala-Gly. FbnP proteins were assayed with four pools of five amino acids (Pool 1: SATPG, Pool 2: VLIMC, Pool 3: NDEQF, Pool 4: YWHKR) and then with the individual amino acids from Pool 1. FbnG was assayed with octanoate, nonanoate, decanoate, and 3-hydroxydecanoate.

\section{ATP-[ $\left.{ }^{32} \mathrm{P}\right] \mathrm{PP} \mathrm{i}_{\mathrm{i}}$ exchange assays to determine A domain specificity of FbnD-S- $\beta-A l a$.} To obtain holo-FbnD, FbnD was overproduced and purified from BL21(DE3) cells that overproduced Sfp (strain BAP1). FbnD-S- $\beta$-Ala was formed in a reaction containing 75 mM Tris-HCl (pH 7.5 at $25^{\circ} \mathrm{C}$ ), $10 \mathrm{mM} \mathrm{MgCl} 2,1 \mathrm{mM}$ TCEP, $3.5 \mathrm{mM}$ ATP, $2 \mu \mathrm{M} \beta$-Ala and $5 \mu \mathrm{M}$ holo-FbnD. This reaction was incubated for 1 hour at $25^{\circ} \mathrm{C}$. Ten-microliters of this reaction was added to standard $A T P-\left[{ }^{32} \mathrm{P}\right] \mathrm{PP}_{\mathrm{i}}$ exchange assays described above where the reactions had $1 \mathrm{mM}$ of $\beta$-Ala, L-Ala, or just water added to the reaction. Repeating the assays with FbnD overproduced and purified from BAP1 or BL21(DE3) strains and then treated with Sfp and CoA to form holo-T domains as previously described ${ }^{5}$ did not have any impact on the observed results.

\section{Radioactive assays of FbnF and FbnN with malonyl-CoA or $(2 R, S)$-methylmalonyl-} CoA. Recognition of either malonyl-CoA or $(2 R, S)$-methylmalonyl-CoA by the AT domains of FbnF and FbnN was tested as previously described ${ }^{6}$. 
L-DOPA Dioxygenase Assay. Supernatants from cultures of E. coli BL21(DE3) overexpressing $f b n L M$ were analyzed with a colorimetric and fluorometric L-DOPA dioxygenase (DOD) assay. Pre-cultures were prepared by growing E. coli BL21(DE3) containing pTEV-fbnLfbnM or pTEV5 overnight at $37^{\circ} \mathrm{C}$. Fresh culture tubes containing $5 \mathrm{~mL}$ LB with carbenicillin $(100 \mathrm{ug} \mathrm{mL}-1)$ were inoculated to $\mathrm{OD}_{600 \mathrm{~nm}}=0.01$. Cultures were incubated at $30{ }^{\circ} \mathrm{C}$ while shaking. Once the $\mathrm{OD}_{600}$ reached $\sim 0.5$, FbnLM overexpression was induced with $100 \mu \mathrm{M}$ IPTG. The cultures grew for another $5 \mathrm{hr}$, and at the end of incubation cells were removed from the cultures by centrifugation and the supernatants were sterilized by filtration.

The $5 X$ DOD reaction buffer was prepared by mixing $5 \mathrm{~mL}$ 10X DOD buffer, $3 \mathrm{~mL}$ water, $0.088 \mathrm{~g} \mathrm{~L}$-ascorbic acid, and $50 \mu \mathrm{L} 100 \mathrm{mM} \mathrm{FeCl}_{2}$. The $\mathrm{pH}$ was adjusted to 6.0 using $5 \mathrm{M}$ $\mathrm{NaOH}$, and water was added to a final volume of $9.5 \mathrm{~mL}$. 10X DOD buffer contains 500 $\mathrm{mM}$ morpholinoethanesulfonic acid (MES) monohydrate, $1 \mathrm{M} \mathrm{NaCl}, 1 \mathrm{M}$ tricine, and 100 $\mathrm{mM}$ glycine. The final 5X DOD reaction master mix was prepared by mixing $237.5 \mu \mathrm{L}$ of the $5 X$ DOD reaction buffer with $12.5 \mu \mathrm{L}$ of $2.5 \mathrm{mg} \mathrm{mL}^{-1} 6 \mathrm{xH}$ is-MBP-L-DOPA dioxygenase in $50 \%(\mathrm{v} / \mathrm{v})$ glycerol with storage buffer. The storage buffer contains $250 \mathrm{mM} \mathrm{NaCl}$ and $10 \mathrm{mM}$ Tric-Cl.

Every sample was assayed by mixing $80 \mu \mathrm{L}$ with $20 \mu \mathrm{L} 5 \mathrm{X}$ DOD reaction master mix and incubating at $30^{\circ} \mathrm{C}$ for up to $16 \mathrm{hr}$. Reactions were monitored by measuring absorbance at $420 \mathrm{~nm}$ and $470 \mathrm{~nm}$ and fluorescence (excitation: $470 \mathrm{~nm}$, emission: $515 \mathrm{~nm}$ ). A standard curve was prepared by adding $1000,500,250,100,50$, and $0 \mu \mathrm{M} \mathrm{L-DOPA}$ to supernatant from E. coli BL21(DE3) containing pTEV5. 
In vitro L-DOPA formation assays. Fifty-microliter reactions contained $100 \mathrm{mM}$ Tris$\mathrm{HCl}\left(\mathrm{pH} 7.5\right.$ at $\left.25^{\circ} \mathrm{C}\right), 100 \mathrm{mM} \mathrm{NaCl}, 20 \mathrm{mM} \mathrm{H}_{2} \mathrm{O}_{2}, 500 \mu \mathrm{M}$ L-Tyr, $2 \mathrm{mM}$ dithiothreitol, 5 $\mu \mathrm{g} \mathrm{FbnL} / \mathrm{M}$. Reactions were run for $30 \mathrm{~min}$ and then stopped by the addition of $15 \mu \mathrm{L}$ of $20 \%(w / v)$ trichloroacetic acid. In vitro assays were analyzed by HPLC with a Shimadzu HPLC system (Shimadzu Co., Columbia, MD, USA) equipped with a fluorescence detector. HPLC separations were performed using a Luna C18 column (250 x $4.6 \mathrm{~mm} ; 5$ $\mu \mathrm{m}$ particle size). The mobile phase was $2.4 \%$ acetonitrile and $0.08 \%$ formic acid in water, the flow rate was $1.0 \mathrm{~mL} \mathrm{~min}^{-1}$, and the injection volume was $2 \mu \mathrm{L}$. L-DOPA and L-tyrosine were detected using the fluorescence detector (excitation: $281 \mathrm{~nm}$, emission: $314 \mathrm{~nm}$ ).

Purification of siderophores FBN A and B. Wild-type A. fabrum C58 was grown in yeast extract mannitol (YEM) media ${ }^{7}$ (mannitol $10 \mathrm{~g}$, yeast extract $1 \mathrm{~g}, \mathrm{~K}_{2} \mathrm{HPO}_{4} 0.5 \mathrm{~g}$, $\mathrm{CaCl}_{2} \cdot \mathrm{H}_{2} \mathrm{O} 0.2 \mathrm{~g}, \mathrm{NaCl} 0.2 \mathrm{~g}$, and $\mathrm{MgSO}_{4} \cdot 7 \mathrm{H}_{2} \mathrm{O} 0.2 \mathrm{~g} \mathrm{~L}^{-1}$ ), with $100 \mu \mathrm{M}$ dipyridyl (Sigma) for 2 days at $28{ }^{\circ} \mathrm{C}$ shaking at 200 RPM. Turbid culture $(1.5 \mathrm{~mL})$, twice washed with tris minimal media adapted for Agrobacteria ${ }^{8}\left(\mathrm{NaCl} 5.8 \mathrm{~g}, \mathrm{KCl} 3.7 \mathrm{~g}, \mathrm{CaCl}_{2} \cdot 2 \mathrm{H}_{2} \mathrm{O} 0.15 \mathrm{~g}\right.$, $\mathrm{MgCl}_{2} \cdot 6 \mathrm{H}_{2} \mathrm{O} 0.1 \mathrm{~g}, \mathrm{NH}_{4} \mathrm{Cl} 1.1 \mathrm{~g}, \mathrm{Na}_{2} \mathrm{SO}_{4} 0.142 \mathrm{~g}, \mathrm{KH}_{2} \mathrm{PO}_{4} 0.272 \mathrm{~g}$, glucose $4 \mathrm{~g}, \mathrm{MnSO}_{4}$ $1 \mu \mathrm{M}$, and tris base $\left.12.1 \mathrm{~g} \mathrm{~L}^{-1}\right)$, was used to inoculate $1 \mathrm{~L}$ tris minimal media with $20 \mu \mathrm{M}$ dipyridyl. Eight $1 \mathrm{~L}$ cultures were incubated in $2.8 \mathrm{~L}$ glass baffled flasks at $28{ }^{\circ} \mathrm{C}$ while shaking at 200 RPM for 6-8 days, depending on presence of siderophore as determined by CAS assay ${ }^{9}$. Cells were pelleted by centrifugation and the supernatant was filtered by vacuum through a $0.2 \mu \mathrm{m}$ filter (Millipore). 
Filtered supernatant was combined with washed HP-20 resin (Sigma) $\left(\sim 70 \mathrm{~g} \mathrm{~L}^{-1}\right.$ of supernatant) and shaken for $4 \mathrm{hr}$ at $25^{\circ} \mathrm{C}$. The HP-20 was then retained by filtration using Miracloth (Millipore), washed with $\mathrm{H}_{2} \mathrm{O}$ and extracted with acetone while stirring for $1 \mathrm{hr}$. The acetone was filtered by gravity through a milk filter and HP-20 was washed with additional acetone. Acetone was removed in vacuo until a small volume remained, then $15 \mathrm{~g}$ of washed HP-20SS were added, contents were mixed, and the remaining acetone was removed. HP-20SS was further dried overnight under high vacuum. Organic compounds bound to the HP-20SS were eluted by column chromatography with a step gradient containing $10,25,50,75$, and $100 \% \mathrm{MeOH}$ in $\mathrm{H}_{2} \mathrm{O}$.

The fractions were analyzed for the compound of interest by analytical RP HPLC-MS (5 min isocratic development at $100 \% \mathrm{H}_{2} \mathrm{O}, 50$ min gradient at $0.5 \mathrm{~mL} \mathrm{~min}^{-1} 10 / 90 \%$ to $33 / 67 \% \mathrm{ACN}-\mathrm{H}_{2} \mathrm{O}$ containing $0.05 \%$ trifluoroacetic acid, and 5 min isocratic development at $100 \% \mathrm{ACN})$ using a Phenomenex Luna C18 column $(250 \times 10 \mathrm{~mm}, 5 \mu \mathrm{m})$ on a Shimadzu Prominence HPLC system and a Micromass LCT mass spectrometer. The elution of metabolites was monitored at $254 \mathrm{~nm}$ and $286 \mathrm{~nm}$. The $50 \%$ fraction contained several related compounds including the putative compound of interest ( $t_{R} 46.5 \mathrm{~min}$ ) which was further purified by semi-preparative RP HPLC fractionation using a Phenomenex Luna C18 column $(250 \times 10 \mathrm{~mm}, 5 \mu \mathrm{M})$ and the aforementioned separation conditions (flow rate was adjusted to $4 \mathrm{~mL} \mathrm{~min}^{-1}$ ) to yield FBN A and FBN B (5.0 mg). These processes were repeated for the supernatant from a culture of a mutant strain containing a deletion in the gene $f b n N$ and the comparable fractions were analyzed by RP HPLC-MS. 
General Experimental Procedures for Structure elucidation. Optical rotations were measured on a Perkin-Elmer 241 Polarimeter. UV spectra were recorded on an Aminco/OLIS UV-Vis Spectrophotometer. IR spectra were measured with a Bruker Equinox 55/S FT-IR Spectrophotometer. 1D and 2D NMR spectra were obtained in DMSO with a Bruker Avance $600 \mathrm{MHz}$ spectrometer with ${ }^{1} \mathrm{H}\left\{{ }^{13} \mathrm{C} /{ }^{15} \mathrm{~N}\right\}$ cryoprobe and 500 $\mathrm{MHz}$ spectrometer with ${ }^{13} \mathrm{C} /{ }^{15} \mathrm{~N}\left\{{ }^{1} \mathrm{H}\right\}$ cryoprobe. HR-ESI-MS and ESI-MS ${ }^{2}$ data were acquired with a Bruker MaXis 4G QTOF mass spectrometer. FT-ICR MS and FT-ICR MS² data were acquired with a Bruker SolariX XR ${ }^{T M}$ FT-ICR mass spectrometer, which is able to reveal the fine structure in isotopic patterns that are specific to the exact molecular formulae of the detected compounds. ESIMS of the $\mathrm{Fe}^{3+}$ complex of FBN A was acquired with a Waters Micromass LCT TOF mass spectrometer.

FBN A and B: colorless oil; [ $\alpha]^{25} \mathrm{D}-20$ (c 0.1, MeOH); UV (MeOH) $\lambda_{\max }(\log \varepsilon) 244$ (3.29) nm, 289 (3.11) nm, 304 (2.75) nm; IR (ATR) $U_{\max }$ 3416, 2927, 2856, 1701, 1635, 1613, 1566, 1476, 1459, 1407, 1372, 1354, 1303, 1267, 1228, 1195, 1134, 1088, 1039, 1021 , $1007 \mathrm{~cm}^{-1} ;{ }^{1} \mathrm{H}$ and ${ }^{13} \mathrm{C}$ NMR (See Table S1, S2); HRESIMS [M] ${ }^{+} \mathrm{m} / \mathrm{z} 1008.4348$ (calculated for $\mathrm{C}_{44} \mathrm{H}_{66} \mathrm{~N}_{9} \mathrm{O}_{16} \mathrm{~S}^{+}, 1008.4343$ ). 

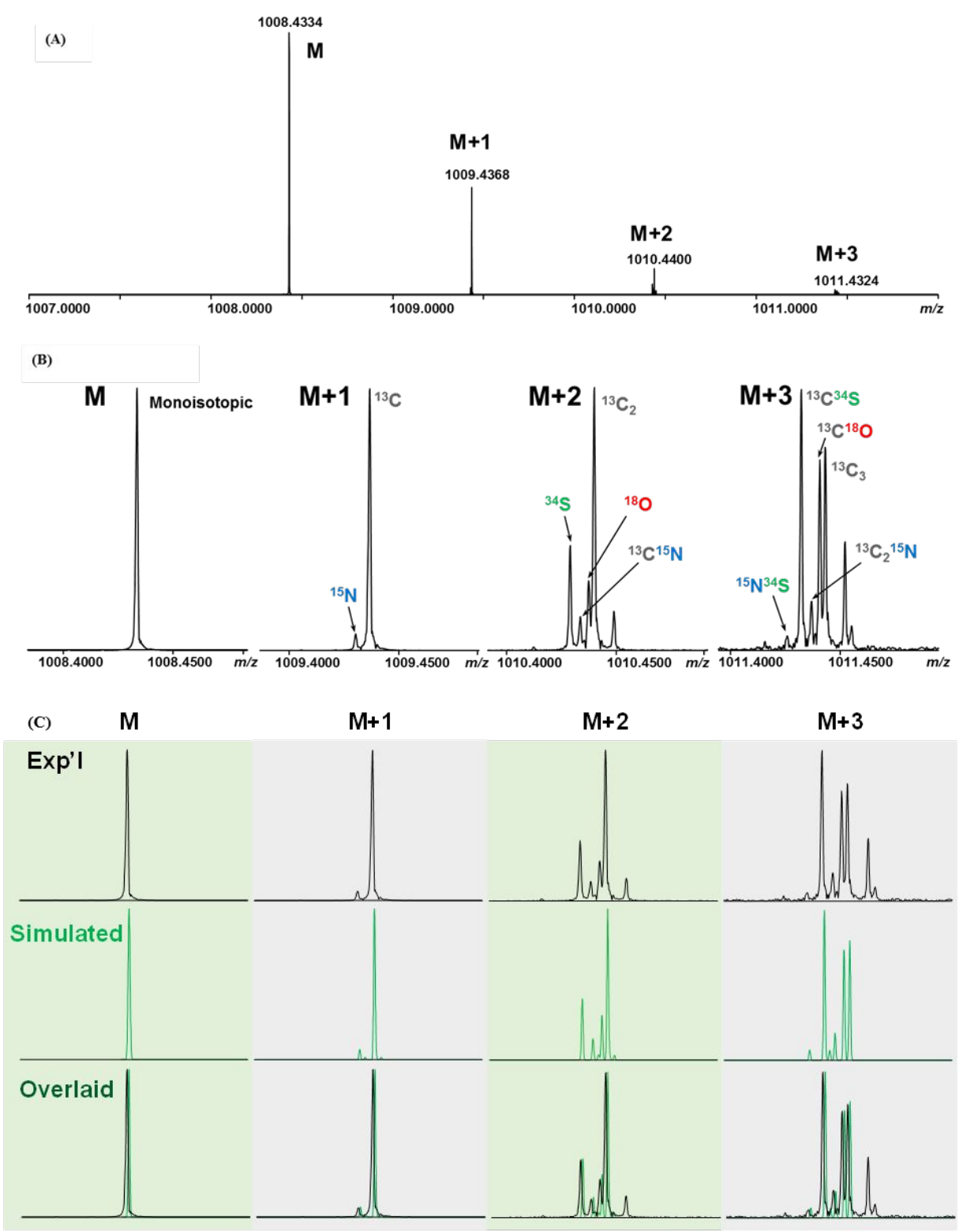

Figure S1 FT-ICR MS of FBN A. (A) The ${ }^{13} \mathrm{C}$ isotopic envelope of the singly charged peptide and the expanded segments. (B) The isotopic fine structure of the $M+1, M+2$, and $\mathrm{M}+3$ isotopic patterns. (C) Comparison between experimental and theoretical isotopic fine structure distributions. 


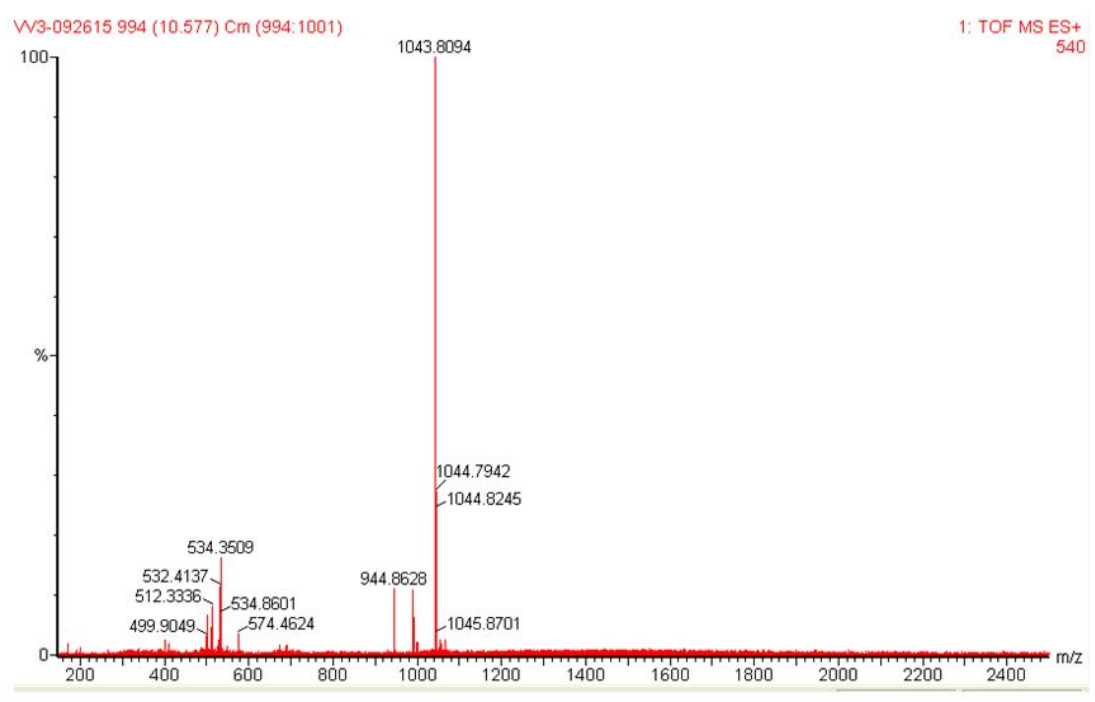

Figure S2 ESI-MS of FBN iron complex formation. ESI-MS showing $\mathrm{m} / \mathrm{z}$ shift of the FBNs when mixed with $\mathrm{FeCl}_{3}$ to produce a 1:1 complex as $\left.\mathrm{M}+\mathrm{Fe}^{3+}-3 \mathrm{H}-\mathrm{H}_{2} \mathrm{O}\right]^{+}$. 
A
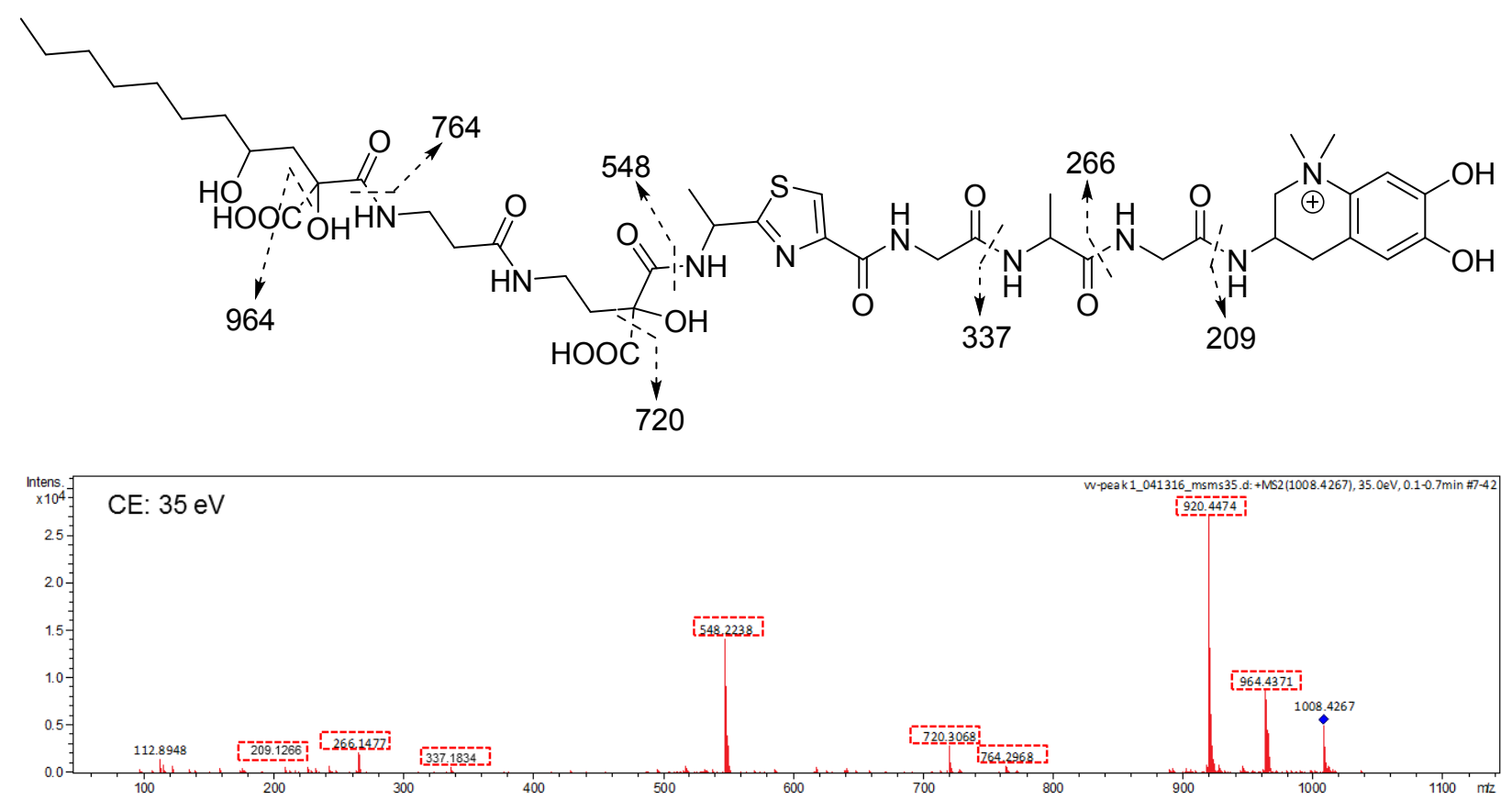

B

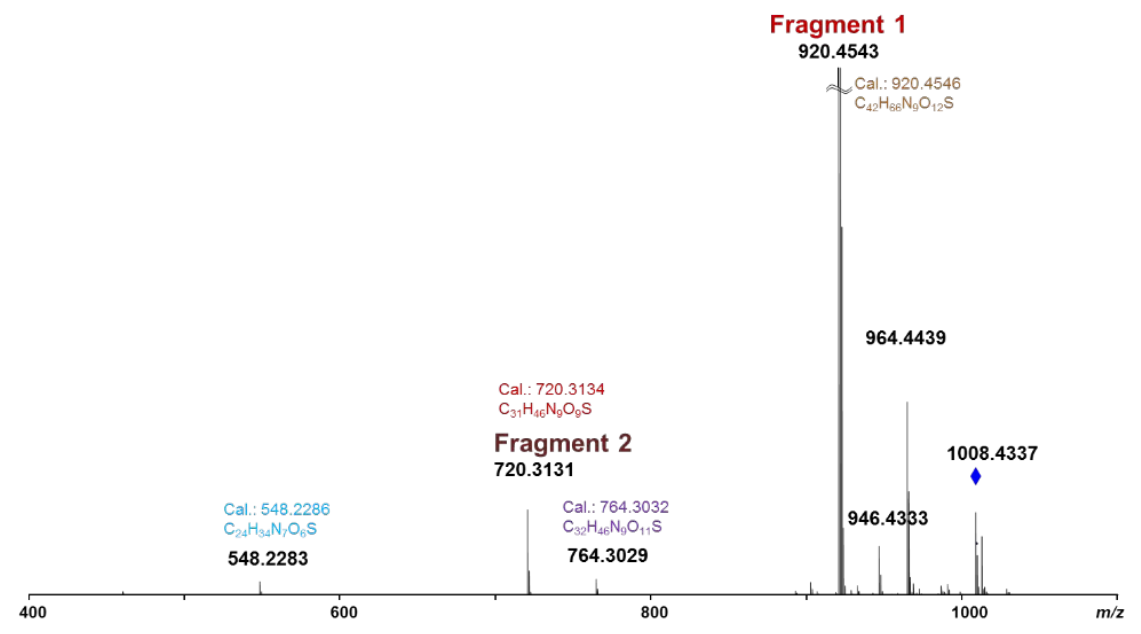

Figure S3 Characteristic fragments in the ESI-MS² spectrum of FBN A and FBN B. A) Fragmentation of FBN A and B observed in the ESI-MS ${ }^{2}$ analysis. B) Fragments 1 and 2 indicate those for which an exact molecular formula was assigned based on fine structure isotopic patterns (data not shown). 

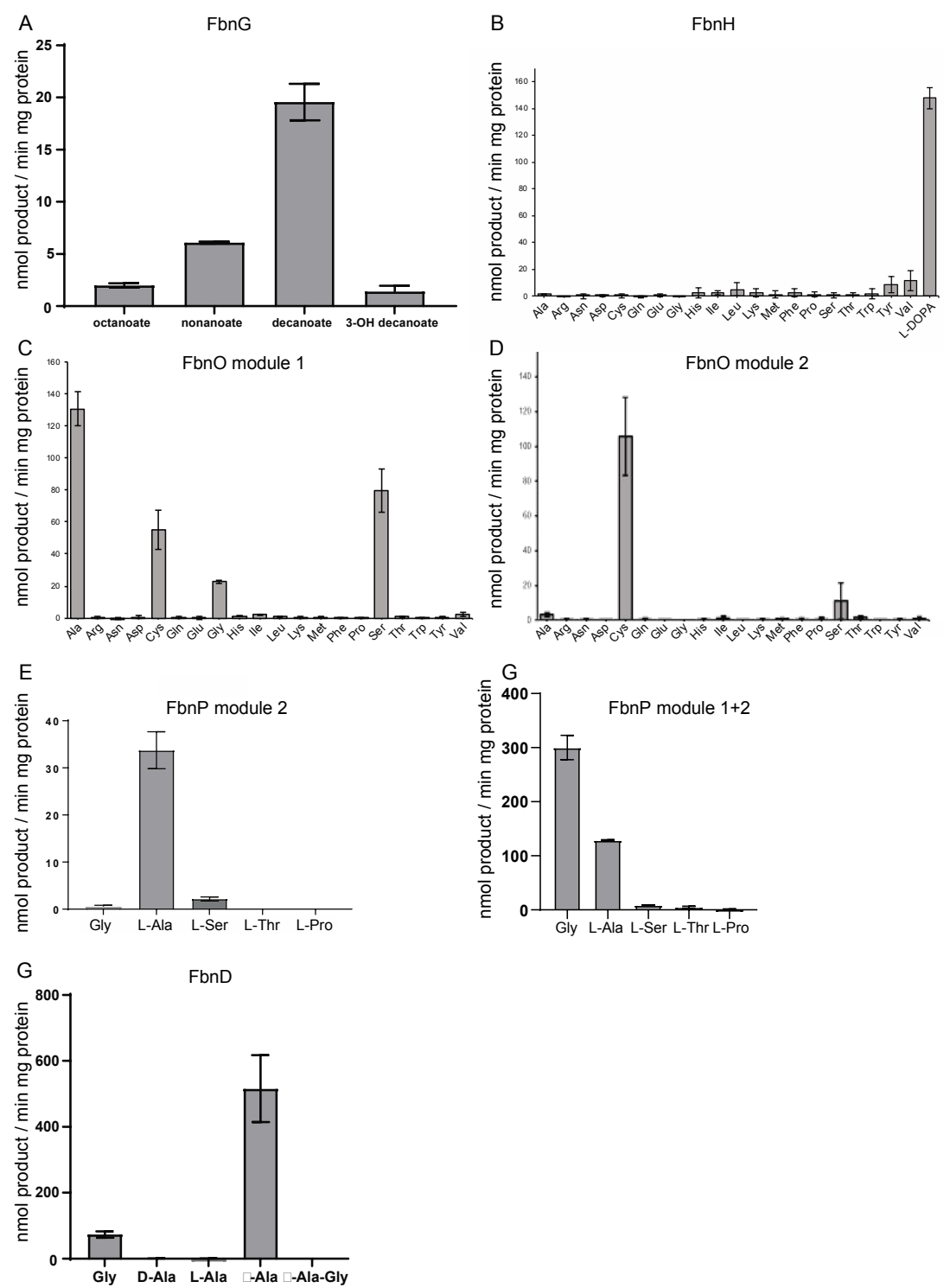

Figure S4 Substrate specificity of NRPS A domains and Acyl-CoA ligase domain of FbnG. Proteins were assayed for their ability to activate select fatty acids (A) and select amino acids $(B-G)$ as determined by $A T P / P P_{i}$ exchange assay. 


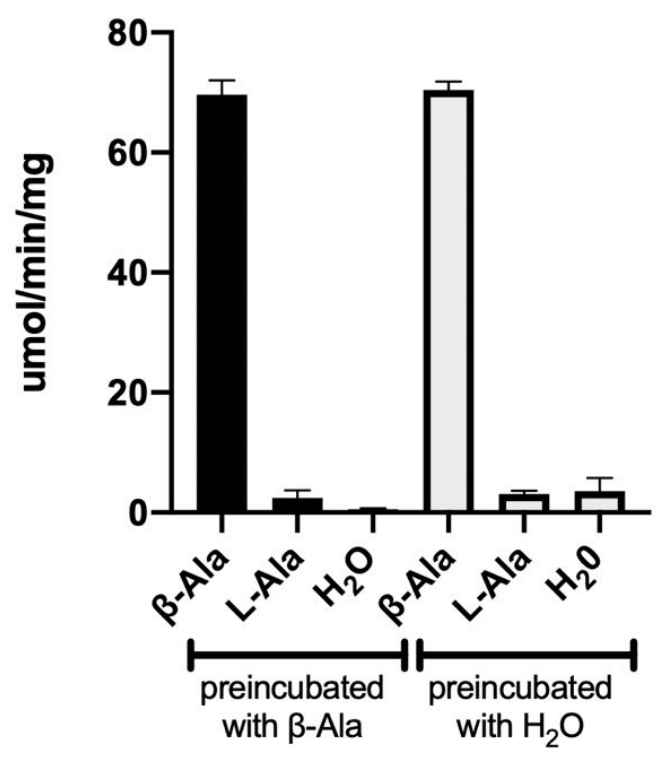

Figure S5 Evaluating FbnD activation of $\beta$-Ala and L-Ala after FbnD-S- $\beta$-Ala formation. ATP/PPi exchange assays monitoring $\beta$-Ala and L-Ala activation by FbnD preincubated with substoichiometric amounts of $\beta$-Ala or no $\beta$-Ala prior to the start of the assay. 

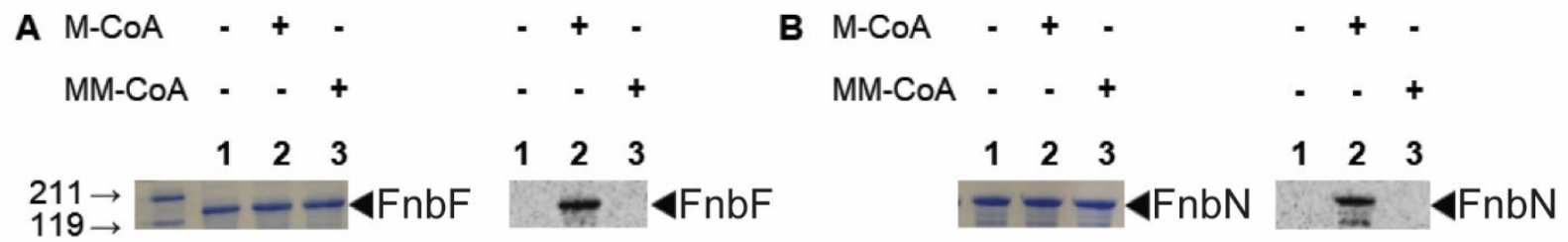

Figure S6 Substrate specificities of the AT domains of FbnF and FbnN. The left side of each panel shows the SDS-PAGE/Coomassie blue analysis of the reaction mixtures; the right side of each panel shows the corresponding phosphorimage. Plus or minus signs indicate the presence or absence, respectively, of the radioactive substrate. $\mathrm{FbnF}(\mathrm{A})$ and $\mathrm{FbnN}(\mathrm{B})$ are shown to be radiolabeled only in the presence of malonyl-CoA. M-CoA, $\left[{ }^{14} \mathrm{C}-\right.$ $\mathrm{C} 2]-$ malonyl-CoA; MM-CoA, $\left[{ }^{14} \mathrm{C}-\mathrm{C} 2\right]-(R, S)-$ methylmalonyl-CoA. 
A.

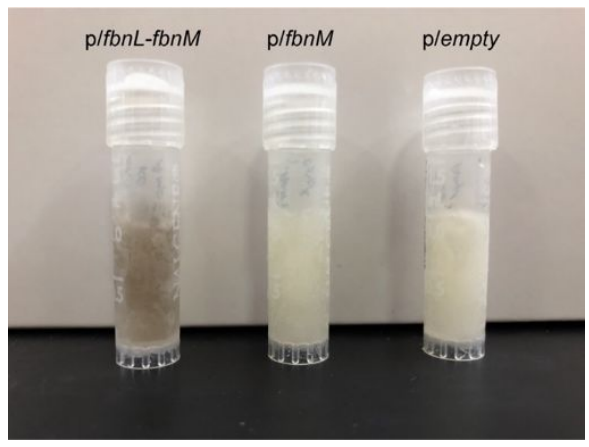

B.

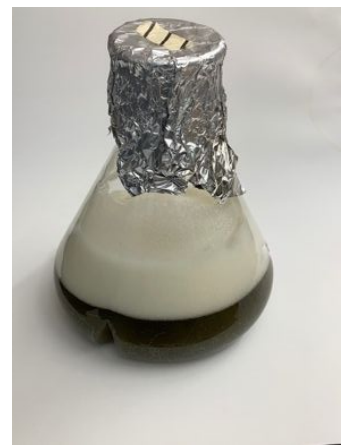

Figure S7 Pigment production by overproduced FbnL/M in E. coli. A) Pigment production was observed only when $f b n L$ and $f b n M$ are expressed together. B) Representative $1 \mathrm{~L}$ $f b n L M$ overexpression culture used to purify FbnL/M. 
Table S1. ${ }^{1} \mathrm{H}$ and ${ }^{13} \mathrm{C}$ NMR data for FBN A $\left(600 \mathrm{MHz},{ }^{1} \mathrm{H} ; 125 \mathrm{MHz},{ }^{13} \mathrm{C}\right.$, DMSO- $\left.d_{6}\right)$.

\begin{tabular}{|c|c|c|c|c|c|c|}
\hline & Position & ${ }^{13} \mathrm{C}$ & ${ }^{1} \mathbf{H}$ & ${ }^{1} \mathrm{H}-{ }^{1} \mathrm{H}$ COSY & НМВС & ${ }^{13} \mathrm{C}-{ }^{13} \mathrm{C}$ COSY \\
\hline \multirow[t]{14}{*}{ Dmaq } & 2 & $\begin{array}{l}64.4 \\
\mathrm{CH}_{2}\end{array}$ & $\begin{array}{l}3.76, \mathrm{~m} \\
3.47, \mathrm{~m}\end{array}$ & $\begin{array}{l}2 \mathrm{~b}, 3 \\
2 \mathrm{a}, 3\end{array}$ & & 3 \\
\hline & 3 & $39.2, \mathrm{CH}$ & $4.45, \mathrm{~m}$ & $2,4,13$ & & 2,4 \\
\hline & 4 & $\begin{array}{l}30.9 \\
\mathrm{CH}_{2}\end{array}$ & $2.82, \mathrm{~m}$ & 3 & $3,5,10$ & 3,5 \\
\hline & 5 & $118.5, \mathrm{C}$ & & & & $4,6,10$ \\
\hline & 6 & $\begin{array}{l}115.4 \\
\mathrm{CH}\end{array}$ & $6.50, \mathrm{~m}$ & & $4,7,8,10$ & 5,7 \\
\hline & 7 & $146.8, \mathrm{C}$ & & & & 6 \\
\hline & 8 & $145.4, \mathrm{C}$ & & & & 9 \\
\hline & 9 & $\begin{array}{l}107.9 \\
\mathrm{CH}\end{array}$ & $7.30, \mathrm{~m}$ & & $4,5,7,8,10$ & 8,10 \\
\hline & 10 & $132.8, \mathrm{C}$ & & & & 5,9 \\
\hline & 11 & $\begin{array}{l}57.9 \\
\mathrm{CH}_{3}\end{array}$ & $3.49, \mathrm{~s}$ & & $2,10,12$ & \\
\hline & 12 & $\begin{array}{l}56.9 \\
\mathrm{CH}_{3}\end{array}$ & $3.57, \mathrm{~s}$ & & $2,10,11$ & \\
\hline & 13 & & $7.98, \mathrm{~d}(7.0)$ & 3 & 3,14 & \\
\hline & 7-OH & & 9.88, br s & & & \\
\hline & 8-OH & & 9.41, br s & & & \\
\hline \multirow[t]{3}{*}{ Gly1 } & 14 & $169.1, \mathrm{C}$ & & & & 15 \\
\hline & 15 & $\begin{array}{l}42.1 \\
\mathrm{CH}_{2}\end{array}$ & $3.76, \mathrm{~m} ; 3.69, \mathrm{~m}$ & 16 & 14,16 & 14 \\
\hline & 16 & & $8.43, \mathrm{~m}$ & 15 & 17 & \\
\hline \multirow[t]{4}{*}{ Ala } & 17 & $172.8, \mathrm{C}$ & & & & 18 \\
\hline & 18 & $49.1, \mathrm{CH}$ & $4.19, \mathrm{~m}$ & 19,20 & 17,19 & 17,19 \\
\hline & 19 & $\begin{array}{l}17.5 \\
\mathrm{CH}_{3}\end{array}$ & $1.26, \mathrm{~d}(6.7)$ & 18 & 17,18 & 18 \\
\hline & 20 & & $8.44, \mathrm{~m}$ & & $18,19,21$ & \\
\hline \multirow[t]{3}{*}{ Gly2 } & 21 & $169.4, \mathrm{C}$ & & & & 22 \\
\hline & 22 & $\begin{array}{l}41.9 \\
\mathrm{CH}_{2}\end{array}$ & $\begin{array}{l}4.05 \text {, dd }(5.3,16) \\
3.88 \text {, dd }(5.0,16)\end{array}$ & 23 & 21,24 & 21 \\
\hline & 23 & & $8.43, \mathrm{~m}$ & 22 & 24 & \\
\hline \multirow[t]{7}{*}{ Ala-Thz } & 24 & $160.9, \mathrm{C}$ & & & & 25 \\
\hline & 25 & $148.8, \mathrm{C}$ & & & & 24,29 \\
\hline & 27 & $174.5, \mathrm{C}$ & & & & 30 \\
\hline & 29 & $\begin{array}{l}124.0 \\
\mathrm{CH}\end{array}$ & $7.88, \mathrm{~s}$ & & 25,27 & 25 \\
\hline & 30 & $47.2, \mathrm{CH}$ & $5.17, \mathrm{dq}(6.8,7.6)$ & 31,32 & 27,31 & 27,31 \\
\hline & 31 & $\begin{array}{l}20.3 \\
\mathrm{CH}_{3}\end{array}$ & $1.56, \mathrm{~d}(6.8)$ & 30 & 27,30 & 30 \\
\hline & 32 & & $8.57, \mathrm{~d}(7.6)$ & 30 & 33 & \\
\hline \multirow[t]{6}{*}{ Ahpa } & 33 & $169.8, \mathrm{C}$ & & & & \\
\hline & 34 & $78.0, \mathrm{C}$ & & & & 35 \\
\hline & 35 & $\begin{array}{l}35.2 \\
\mathrm{CH}_{2}\end{array}$ & $2.07, \mathrm{~m}$ & 36 & $34,36,37$ & 34 \\
\hline & 36 & $\begin{array}{l}34.2 \\
\mathrm{CH}_{2}\end{array}$ & $\begin{array}{l}3.13, \mathrm{~m} \\
3.04, \mathrm{~m}\end{array}$ & 35,38 & & \\
\hline & 37 & $171.9, \mathrm{C}$ & & & & \\
\hline & 38 & & $7.87, \mathrm{~m}$ & 36 & 36,38 & \\
\hline \multirow[t]{2}{*}{$\beta$-Ala } & 39 & $170.3, \mathrm{C}$ & & & & 40 \\
\hline & 40 & $\begin{array}{l}34.8 \\
\mathrm{CH}_{2}\end{array}$ & $2.28, \mathrm{~m}$ & 41 & 39,41 & 39 \\
\hline
\end{tabular}




\begin{tabular}{|c|c|c|c|c|c|c|}
\hline & 41 & 35.4 , & $3.31, \mathrm{~m}$ & 40,42 & 39,40 & \\
\hline \multirow{13}{*}{ Cdua } & 42 & & $8.15, \mathrm{~m}$ & 41 & 41,43 & \\
\hline & 43 & 169.8, C & & & & \\
\hline & 44 & 77.6, C & & & & 45 \\
\hline & 45 & $\begin{array}{l}40.7 \\
\mathrm{CH}_{2}\end{array}$ & $2.32, \mathrm{~m}$ & 46 & $\begin{array}{l}43,44,47, \\
54\end{array}$ & 44 \\
\hline & 46 & $78.1, \mathrm{CH}$ & $4.60, \mathrm{~m}$ & 45,47 & 48 & 47 \\
\hline & 47 & $\begin{array}{l}34.5 \\
\mathrm{CH}_{2}\end{array}$ & $1.69, \mathrm{~m} ; 1.60, \mathrm{~m}$ & 46,48 & 48,49 & 46 \\
\hline & 48 & $\begin{array}{l}24.7 \\
\mathrm{CH}_{2}\end{array}$ & $1.34, \mathrm{~m}$ & 47 & 49 & 49 \\
\hline & 49 & $\begin{array}{l}28.8 \\
\mathrm{CH}_{2}\end{array}$ & $1.29, \mathrm{~m}$ & & 50,51 & 48 \\
\hline & 50 & $\begin{array}{l}28.7 \\
\mathrm{CH}_{2}\end{array}$ & $1.29, \mathrm{~m}$ & & 49,51 & 51 \\
\hline & 51 & $\begin{array}{l}31.3, \\
\mathrm{CH}_{2}\end{array}$ & $1.24, \mathrm{~m}$ & & 50,52 & 50 \\
\hline & 52 & $\begin{array}{l}22.1 \\
\mathrm{CH}_{2}\end{array}$ & $1.28, \mathrm{~m}$ & 51 & 51,53 & 53 \\
\hline & 53 & $\begin{array}{l}14.0 \\
\mathrm{CH}_{3}\end{array}$ & $0.86, \mathrm{t}(6.6)$ & 52 & 51,52 & 52 \\
\hline & 54 & 173.9, C & & & & \\
\hline
\end{tabular}


Table S2. ${ }^{1} \mathrm{H}$ and ${ }^{13} \mathrm{C}$ NMR data for FBN B $\left(600 \mathrm{MHz},{ }^{1} \mathrm{H} ; 125 \mathrm{MHz},{ }^{13} \mathrm{C}\right.$, DMSO- $\left.d_{6}\right)$.

\begin{tabular}{|c|c|c|c|c|c|c|}
\hline & Position & ${ }^{13} \mathrm{C}$ & ${ }^{1} \mathbf{H}$ & ${ }^{1} \mathrm{H}-{ }^{1} \mathrm{H}$ COSY & НМВС & ${ }^{13} \mathrm{C}-{ }^{13} \mathrm{C}$ COSY \\
\hline \multirow[t]{14}{*}{ Dmaq } & 2 & $\begin{array}{l}64.4 \\
\mathrm{CH}_{2}\end{array}$ & $\begin{array}{l}3.76, \mathrm{~m} \\
3.47, \mathrm{~m}\end{array}$ & $\begin{array}{l}2 \mathrm{~b}, 3 \\
2 \mathrm{a}, 3\end{array}$ & & 3 \\
\hline & 3 & $39.2, \mathrm{CH}$ & $4.45, \mathrm{~m}$ & $2,4,13$ & & 2,4 \\
\hline & 4 & $\begin{array}{l}30.9 \\
\mathrm{CH}_{2}\end{array}$ & $2.82, \mathrm{~m}$ & 3 & $3,5,10$ & 3,5 \\
\hline & 5 & $118.5, \mathrm{C}$ & & & & $4,6,10$ \\
\hline & 6 & $\begin{array}{l}115.4 \\
\mathrm{CH}\end{array}$ & $6.50, \mathrm{~m}$ & & $4,7,8,10$ & 5,7 \\
\hline & 7 & $146.8, \mathrm{C}$ & & & & 6 \\
\hline & 8 & $145.4, \mathrm{C}$ & & & & 9 \\
\hline & 9 & $\begin{array}{l}107.9 \\
\mathrm{CH}\end{array}$ & $7.30, \mathrm{~m}$ & & $4,5,7,8,10$ & 8,10 \\
\hline & 10 & $132.8, \mathrm{C}$ & & & & 5,9 \\
\hline & 11 & $\begin{array}{l}57.9 \\
\mathrm{CH}_{3}\end{array}$ & $3.49, \mathrm{~s}$ & & $2,10,12$ & \\
\hline & 12 & $\begin{array}{l}56.9 \\
\mathrm{CH}_{3}\end{array}$ & $3.57, \mathrm{~s}$ & & $2,10,11$ & \\
\hline & 13 & & $7.98, \mathrm{~d}(7.0)$ & 3 & 3,14 & \\
\hline & 7-OH & & 9.88, br s & & & \\
\hline & $8-\mathrm{OH}$ & & 9.41 , br s & & & \\
\hline \multirow[t]{3}{*}{ Gly1 } & 14 & $169.1, \mathrm{C}$ & & & & 15 \\
\hline & 15 & $\begin{array}{l}42.1 \\
\mathrm{CH}_{2}\end{array}$ & $3.76, \mathrm{~m} ; 3.69, \mathrm{~m}$ & 16 & 14,16 & 14 \\
\hline & 16 & & $8.43, \mathrm{~m}$ & 15 & 17 & \\
\hline \multirow[t]{4}{*}{ Ala } & 17 & $172.8, \mathrm{C}$ & & & & 18 \\
\hline & 18 & 49.1, CH & $4.19, \mathrm{~m}$ & 19,20 & 17,19 & 17,19 \\
\hline & 19 & $\begin{array}{l}17.5 \\
\mathrm{CH}_{3}\end{array}$ & $1.26, \mathrm{~d}(6.7)$ & 18 & 17,18 & 18 \\
\hline & 20 & & $8.44, \mathrm{~m}$ & & $18,19,21$ & \\
\hline \multirow[t]{3}{*}{ Gly2 } & 21 & $169.4, \mathrm{C}$ & & & & 22 \\
\hline & 22 & $\begin{array}{l}41.9 \\
\mathrm{CH}_{2}\end{array}$ & $\begin{array}{l}4.05 \text {, dd }(5.3,16) \\
3.88 \text {, dd }(5.0,16)\end{array}$ & 23 & 21,24 & 21 \\
\hline & 23 & & $8.43, \mathrm{~m}$ & 22 & 24 & \\
\hline \multirow[t]{7}{*}{ Ala-Thz } & 24 & $160.9, \mathrm{C}$ & & & & 25 \\
\hline & 25 & $148.8, \mathrm{C}$ & & & & 24,29 \\
\hline & 27 & $174.5, \mathrm{C}$ & & & & 30 \\
\hline & 29 & $\begin{array}{l}124.0 \\
\mathrm{CH}\end{array}$ & $7.88, \mathrm{~s}$ & & 25,27 & 25 \\
\hline & 30 & $47.2, \mathrm{CH}$ & $5.17, \mathrm{dq}(6.8,7.6)$ & 31,32 & 27,31 & 27,31 \\
\hline & 31 & $\begin{array}{l}20.3 \\
\mathrm{CH}_{3}\end{array}$ & $1.56, \mathrm{~d}(6.8)$ & 30 & 27,30 & 30 \\
\hline & 32 & & $8.57, \mathrm{~d}(7.6)$ & 30 & 33 & \\
\hline \multirow[t]{6}{*}{ Ahpa } & 33 & $169.8, \mathrm{C}$ & & & & \\
\hline & 34 & $78.0, \mathrm{C}$ & & & & 35 \\
\hline & 35 & $\begin{array}{l}35.2 \\
\mathrm{CH}_{2}\end{array}$ & $2.07, \mathrm{~m}$ & 36 & $34,36,37$ & 34 \\
\hline & 36 & $\begin{array}{l}34.2 \\
\mathrm{CH}_{2}\end{array}$ & $\begin{array}{l}3.13, \mathrm{~m} \\
3.04, \mathrm{~m}\end{array}$ & 35,38 & & \\
\hline & 37 & $171.9, \mathrm{C}$ & & & & \\
\hline & 38 & & $7.87, \mathrm{~m}$ & 36 & 36,38 & \\
\hline \multirow[t]{4}{*}{ Ala } & 39 & $170.3, \mathrm{C}$ & & & & \\
\hline & 40 & $50.2, \mathrm{CH}$ & $3.91, \mathrm{~m}$ & 41 & & 41 \\
\hline & 41 & $\begin{array}{l}16.8 \\
\mathrm{CH}_{3}\end{array}$ & $1.26, \mathrm{~m}$ & 40,42 & & 40 \\
\hline & 42 & & $8.15, \mathrm{~m}$ & 41 & & \\
\hline \multirow[t]{2}{*}{ Cdua } & 43 & $169.8, \mathrm{C}$ & & & & \\
\hline & 44 & $77.6, \mathrm{C}$ & & & & 45 \\
\hline
\end{tabular}




$\begin{array}{llllll}45 & 40.7, & 2.32, \mathrm{~m} & 46 & 43,44,47, & 44 \\ & \mathrm{CH}_{2} & & & 54 & \\ 46 & 78.1, \mathrm{CH} & 4.60, \mathrm{~m} & 45,47 & 48 & 47 \\ 47 & 34.5, & 1.69, \mathrm{~m} ; 1.60, \mathrm{~m} & 46,48 & 48,49 & 46 \\ & \mathrm{CH}_{2} & & & & \\ 48 & 24.7, & 1.34, \mathrm{~m} & 47 & 49 & 49 \\ & \mathrm{CH}_{2} & & & & \\ 49 & 28.8, & 1.29, \mathrm{~m} & 50,51 & 48 \\ & \mathrm{CH}_{2} & & & \\ 50 & 28.7, & 1.29, \mathrm{~m} & 49,51 & 51 \\ & \mathrm{CH}_{2} & & & 50,52 & 50 \\ 51 & 31.3, & 1.24, \mathrm{~m} & & & \\ 52 & \mathrm{CH}_{2} & & & 51,53 & 53 \\ & 22.1, & 1.28, \mathrm{~m} & 51 & & \\ 53 & \mathrm{CH}_{2} & & & 51,52 & 52 \\ 54 & 14.0, & 0.86, \mathrm{t}(6.6) & 52 & & \end{array}$


Table S3. Predicted functions of ORFs in the fbn gene cluster.

\begin{tabular}{|c|c|c|c|}
\hline $\begin{array}{l}\text { GenBank } \\
\text { Gene } \\
\text { Notationa }\end{array}$ & Fbn gene & Protein ID & Putative Function \\
\hline$\overline{\text { panD }}$ & $f b n A$ & NP_356949.1 & Aspartate $\alpha$-decarboxylase \\
\hline Atu3668 & $f b n B$ & NP_356948.1 & IscR family transcriptional regulator \\
\hline Atu3669 & $f b n C$ & NP_356947.2 & MATE family multidrug efflux pump \\
\hline Atu3670 & $f b n D$ & NP_356946.2 & $\operatorname{NRPS}\left(C-A_{\beta-A l a}-T\right)$ \\
\hline Atu3671 & fbnE & NP_356945.1 & Flavin-dependent monooxygenase \\
\hline Atu3672 & $f b n F$ & NP_356944.1 & PKS (KS-AT $\left.T_{m a l}-K R-T\right)$ \\
\hline Atu3673 & $f b n G$ & NP_356943.2 & PKS (FAAL-T) \\
\hline Atu3675 & $\mathrm{fbnH}$ & NP_356942.1 & NRPS $\left(C-A_{D O P A}-T-R e d\right)$ \\
\hline Atu3676 & fbnl & NP_356941.1 & SAM-dependent methyltransferase \\
\hline Atu3677 & fbnJ & NP_356940.1 & Glutamate-1-semialdehyde aminotransferase \\
\hline Atu3678 & fbnK & NP 356939.2 & MbtH-like protein \\
\hline Atu3679 & $f b n L$ & NP_356938.1 & Heme peroxidase-associated protein \\
\hline Atu3680 & $f b n M$ & NP_356937.2 & Heme peroxidase \\
\hline Atu3681 & $f b n N$ & NP_356936.2 & PKS (KS-AT mal-KR-T) \\
\hline Atu3682 & $\mathrm{fbnO}$ & NP_356935.1 & NRPS (C-A Ala-T-Cy-ACys $-T)$ \\
\hline Atu3683 & $f b n P$ & NP_356934.2 & NRPS $\left(C-A_{G l y}-T-C-A_{A l a}-T-E\right)$ \\
\hline Atu3684 & $f b n Q$ & NP_356933.1 & NRPS (C-T) \\
\hline Atu3685 & $f b n R$ & NP_356932.2 & Type II Thioesterase \\
\hline Atu3686 & fbns & NP_356931.1 & Ferric iron reductase protein \\
\hline Atu3687 & $f b n T$ & NP_356930.2 & TonB-dependent receptor \\
\hline Atu3688 & $f b n U$ & NP_356929.2 & $A B C$ transporter permease \\
\hline Atu3689 & $f b n V$ & NP_356928.1 & $A B C$ transporter permease \\
\hline Atu3690 & $f b n W$ & NP_356927.2 & $A B C$ transporter permease \\
\hline Atu3691 & $f b n X$ & NP_356926.1 & ABC transporter ATPase \\
\hline Atu3692 & $f b n Y$ & NP_356925.2 & FecE-like sigma factor \\
\hline Atu3693 & $f b n Z$ & NP_356924.1 & FecR-like two component sensor kinase \\
\hline
\end{tabular}

${ }^{a}$ GenBank gene notation was assigned by Wood et al. ${ }^{10}$. 
Table S4. Kinetic parameters of FbnO-1 for activation of select amino acid substrates.

\begin{tabular}{cccc}
\hline Amino acid & $K_{\mathrm{m}}(\mathrm{mM})^{a}$ & $V_{\max }(\mathrm{nmol}$ product $/ \mathrm{min} / \mathrm{mg}$ protein $)$ & $V_{\max } / K_{m}$ \\
\hline L-Alanine & $0.38 \pm 0.03$ & $121.57 \pm 3.57$ & 318 \\
L-Cysteine & $5.98 \pm 0.92$ & $91.96 \pm 8.39$ & 15 \\
L-Glycine & $22.20 \pm 3.96$ & $120.78 \pm 12.49$ & 5 \\
L-Serine & $5.99 \pm 0.46$ & $137.79 \pm 4.05$ & 23 \\
\hline
\end{tabular}

${ }^{a}$ The values are from the Michaelis-Menten curve, fit to the results of three independent assays with the standard errors from the nonlinear regression 
Table S5. Proposed Dmaq-containing siderophore BGC locus tags shown in Figure 5.

Species
Locus Tag

5 ' end gene
Locus Tag

3 ' end gene
A. fabrum strain C58

A. fabrum 12D13a

A. fabrum 1D132a

A. fabrum ATCC $31749^{a}$

A. fabrum ARqua1 ${ }^{a}$

A. fabrum Arqua ${ }^{a}$

Rhizobium sp. PDC82a

A. tumefaciens $15955^{a}$

A. tumefaciens $15-174^{a}$

A. tumefaciens CFBP5877a

A. tumefaciens CFBP5499a

A. tumefaciens LBA4404a

A. tumefaciens $\mathrm{F}^{\mathrm{a}}$

Rhizobium sp. NFIX01a

Rhizobium sp. NFIX02a

Rhizobium sp. UGM030330-04a

A. rhizogenes BGGC3284

M. pamukkalensis PK2

R. rhizosphaerae RD15

S. kummerowiae CCBAU 25048

Agrobacterium sp. a22-2

R. oryziradicis N19

A. vitis $\mathrm{AV} 25 / 95^{b}$

A. vitis $\mathrm{SZ}{ }^{b}$

A. vitis $\mathrm{AB}^{b}$

A. vitis $\mathrm{AB}^{b}$

A. tumefaciens $15-172^{b}$

A. tumefaciens 1D1609b

R. rhizosphaerae $\mathrm{MH} 17^{b}$

Atu3670 (FbnD)
Ga0399242_02_681708_685094
Ga0398321_02_718220_721606
Ga0347210_02_691421_694807
HI842_18770
EXN51_RS18715
Ga0115500_2830
Ga0398022_03_301123_304509
Ga0340186_27_47785_51171
Ga0399375_02_860168_863551
Ga0398586_02_859197_862580
EN41_04020
Ga0077515_1646
Ga0059025_03355
Ga0059026_03353
N434DRAFT_04040
EXN68_RS23280
A6302_RS02085
BTR14_RS05860
GR128_RS09690
HFC70_RS13395
BJF95_15780
G6L27_RS24135
BBL07_RS13815
BBI12_RS15030
BBI04_RS14510
Ga0338076_45_294809_295555
Ga0272068_4194
Ga0308746_141154
Ga

Atu3685 (FbnR)

Ga0399242_02_722712_723458

Ga0398321_02_759229_759975

Ga0347210_02_732431_733177

HI842_18705

EXN51_RS18790

Ga0115500_2816

Ga0398022_03_262752_263495

Ga0340186_27_9417_10160

Ga0399375_02_901209_901955

Ga0398586_02_901129_901905

EN41_03945

Ga0077515_16420

Ga0059025_03341

Ga0059026_03339

N434DRAFT_04026

EXN68_RS23355

A6302_RS02005

BTR14_RS05795

GR128_RS09765

HFC70_RS13395

BJF95_15845

G6L27_RS24070

BBL07_RS1745

BBI12_RS15100

BBI04_RS14580

Ga0338076_45_257950_264471

Ga0272068_4207

Ga0308746_141141 


$\begin{array}{lll}\text { Aquamicrobium sp. LC103 } & \text { XW59_RS10960 } & \text { XW59_RS10885 } \\ \begin{array}{l}\text { P. aquimaris KCTC23043 } \\ \text { P. sp. S12M18 }\end{array} & \text { AKU94_RS09275 } & \text { AKU94_RS09190 } \\ \begin{array}{l}\text { Anabaena cylindrica PCC7122 } \\ \text { Acaryochloris marina }\end{array} & \text { AOK75_RS08355 } & \text { EOK75_RS08275 } \\ \text { MBIC11017 } & \text { AM1_A0226 } & \text { Anacy_5452 } \\ \text { Nostoc sp. 'Lobaria pulmonaria' } & \text { NLP_RS01445 } & \text { AM1_A0246 } \\ \begin{array}{l}\text { Nostoc sp. PCC7120 } \\ \text { Chrocosphaera subtropica }\end{array} & \text { All2650 } & \\ \begin{array}{l}\text { ATCC51142 } \\ \text { Gloeocapsasp. PCC7428 }\end{array} & \text { Glo7482_5008 } & \text { NLP_RS01560 } \\ \text { Nodularia spumigena CCY9414 } & \text { N9414_20950 } & \text { All2635 } \\ \begin{array}{l}\text { Phormidesmis priestleyi Ana } \\ \text { Planctomycetaceae isolate }\end{array} & \text { HLUCCA11_05230 } & \text { CCE_3082 } \\ \text { SP176 } & \text { CMJ77_14245 } & \\ \end{array}$

a Strains included in the Group I A. tumefaciens complex in Fig. 5

${ }^{b}$ Strains included in the Group I that are similar to A. vitis AV25/95 in Fig. 5 
Table S6. Primers used for plasmid construction.

\begin{tabular}{|c|c|}
\hline Primer & Sequence $\left(5^{\prime} \rightarrow 3^{\prime}\right)$ \\
\hline 3670 F Ndel & AGGATTTGCGCATATGATGTCGAATGCC \\
\hline 3670 R HindIII & AGCGGAAAGCTTCAACTGGAACAGG \\
\hline 3672 F Ndel & CCGCGCGGCAGCCATATGAACGTAAAACAACAGGCC \\
\hline 3672 R HindIII & GAGTGCGGCCGCAAGCTTTCAACGACCGGCCTCGACGTC \\
\hline 3673 F Ndel & GGAGTTTTTCAGCATATGCAGATCATACCG \\
\hline 3673 R Sacl & TTCATCGGCGATGAGCTCCGATCATCGATT \\
\hline 3675 F Ndel & CCGCGCGGCAGCCATATGAATTACATCGAGCCAGTAGAG \\
\hline 3675 R HindIII & GAGTGCGGCCGCAAGCTTTCATCGTTCACCCTGCTTTCG \\
\hline 8 Duet F & GGAGATATACCATGAGTTCCCAGAC \\
\hline 8 Duet $\mathrm{R}$ & CGCAAGCTTCTATTCTTGCGC \\
\hline 3681 F Ndel & AGCCATATGTCCGACTATACCAGCGCT \\
\hline 3681 R HindIII & GAGTGCGGCCGCAAGCTTTCATCGTGCTTCTGCTCCTCC \\
\hline 3682-1 F Ndel & CCGCGCGGCAGCCATATGATGAACGAATTCTACGCCAAAC \\
\hline 2-1 R HindIII & GAGTGCGGCCGCAAGCTTTCAAAGCCGGTT1 \\
\hline 3682-2 F Asel & GCCGCGCGGCAGCATTAATCTGGCAAACCGGCTTGAGAAT \\
\hline 3682-2 R Xhol & GGTGGTGGTGGTGGTGCTCGAGCTACTGGTCCTTTTGGCCGG \\
\hline 3683-1 F Ncol & GACCCCATGGACAAGACGGAGCATGCGGC \\
\hline 3683-1 R Sall & GGTCATGTCGACCAGAGCATCGACAGC \\
\hline 3-2 F Ncol & AAAACCATGGGGCAATGGAACGAGACGG \\
\hline 3683-2 R HindIII & CAGTGCTTTCCACGTG \\
\hline
\end{tabular}


Table S7. Strains used in this study.

\begin{tabular}{|c|c|c|}
\hline Strain & Purpose or characteristics & Source \\
\hline E. coli DH5a & $\begin{array}{l}\text { fhuA2, } \triangle(\arg F-l a c Z) \cup 169, \text { phoA, } \\
\text { glnV44, } \$ 80, \Delta(\operatorname{lacZ}) M 15, \text { gyrA96, } \\
\text { recA1, relA1, endA1, thi-1, hsdR17 }\end{array}$ & Laboratory strain \\
\hline $\begin{array}{l}\text { E. coli } \\
\text { BL21(DE3)ybdZ::aac(3)-IV }\end{array}$ & $\begin{array}{l}\text { BL21 (DE3) with ybdZ-disrupting } \\
\text { apramycin resistance cassette }\end{array}$ & 4 \\
\hline E. coli S17-1 & $\begin{array}{l}\text { recA thi pro hsdR- } M^{+} \text {RP4-2 Tcr :: } \\
\text { Mu Km :: Tn7 }\end{array}$ & Laboratory strain \\
\hline A. fabrum str.C58 & Wild-type & Laboratory strain \\
\hline A. fabrum str.C58 $\triangle \mathrm{fbnN}$ & C58 with markerless deletion of $f b n N$ & This study \\
\hline
\end{tabular}


Table S8. Plasmids used in this study.

\begin{tabular}{|l|l|l|}
\hline Plasmid & Purpose & Source \\
\hline pACYC duet-1 & T7 coexpression vector & Novagen \\
\hline pACYC duet-fbnK & Coexpression of $f b n K$ & This study \\
\hline pET28b & T7 expression vector & Novagen \\
\hline pET28b-fbnD & Overexpression of $f b n D$ & This study \\
\hline pET28b-fbnF & Overexpression of $f b n F$ & This study \\
\hline pET28b-fbnG & Overexpression of $f b n G$ & This study \\
\hline pET28b-fbnH & Overexpression of $f b n H$ & This study \\
\hline pET28b-fbnN & Overexpression of $f b n N$ & This study \\
\hline pET28b-fbnO-1 & Overexpression of $f b n O$ module 1-coding & This study \\
\hline pET28b-fbnO-2 & Overexpression of $f b n D$ module 2-coding & This study \\
\hline pET28b-fbnP & Overexpression of $f b n P$ & This study \\
\hline pET28b-fbnP-2 & Overexpression of $f b n P$ module 2-coding & This study \\
\hline pBBR1MCS-2 & Broad host range gene expression & 2 \\
\hline pBBR1MCS-2-fbnN & Complementation of $f b n N$ deletion strain & This study \\
\hline pK18mobsacB & Host chromosome integration & ATCC87097 \\
\hline pK18mobsacB-fbnN & Markerless deletion of $f b n N$ & This study \\
\hline pANT841 & high-copy vector for cloning & 11 \\
\hline pTEV841-fbnLfbnM & Overexpression of $f b n L$ and $f b n M$ & This study \\
\hline
\end{tabular}




\section{References}

[1] McMahon, M. D., Rush, J. S., and Thomas, M. G. (2012) Analyses of MbtB, MbtE, and MbtF suggest revisions to the mycobactin biosynthesis pathway in Mycobacterium tuberculosis, J. Bacteriol. 194, 2809-2818.

[2] Kovach, M. E., Elzer, P. H., Hill, D. S., Robertson, G. T., Farris, M. A., Roop, R. M., and Peterson, K. M. (1995) Four new derivatives of the broad-host-range cloning vector pBBR1MCS, carrying different antibiotic-resistance cassettes, Gene 166, 175-176.

[3] Mersereau, M., Pazour, G. J., and Das, A. (1990) Efficient transformation of Agrobacterium tumefaciens by electroporation, Gene 90, 149-151.

[4] Felnagle, E. A., Barkei, J. J., Park, H., Podevels, A. M., McMahon, M. D., Drott, D. W., and Thomas, M. G. (2010) Mbth-like proteins as integral components of bacterial nonribosomal peptide synthetases, Biochemistry 49, 8815-8817.

[5] Schomer, R. A., and Thomas, M. G. (2017) Characterization of the functional variance in MbtHlike protein interactions with a nonribosomal peptide synthetase, Biochemistry 56, 53805390.

[6] Chan, Y. A., and Thomas, M. G. (2010) Recognition of (2S)-aminomalonyl-acyl carrier protein (ACP) and (2R)-hydroxymalonyl-ACP by acyltransferases in zwittermicin A biosynthesis, Biochemistry 49, 3667-3677.

[7] Vincent, J. M. (1970) A manual for the practical study of root nodule bacteria, Blackwell Scientific Publications, Oxford, UK.

[8] Leong, S. A., and Neilands, J. B. (1982) Siderophore production by phytopathogenic microbial species, Arch. Biochem. Biophys. 218, 351-359.

[9] Alexander, D. B., and Zuberer, D. A. (1991) Use of the chrome azurol S reagents to evaluate siderophore production by rhizosphere bacteria, Biol. Fertil. Soils 12, 39-45.

[10] Wood, D. W., Setubal, J. C., Kaul, R., Monks, D. E., Kitajima, J. P., Okura, V. K., Zhou, Y., Chen, L., Wood, G. E., Almeida, N. F., Woo, L., Chen, Y., Paulsen, I. T., Eisen, J. A., Karp, P. D., Bovee, D., Chapman, P., Clendenning, J., Deatherage, G., Gillet, W., Grant, C., Kutyavin, T., Levy, R., Li, M. J., McClelland, E., Palmieri, A., Raymond, C., Rouse, G., Saenphimmachak, C., Wu, Z., Romero, P., Gordon, D., Zhang, S., Yoo, H., Tao, Y., Biddle, P., Jung, M., Krespan, W., Perry, M., Gordon-Kamm, B., Liao, L., Kim, S., Hendrick, C., Zhao, Z. Y., Dolan, M., Chumley, F., Tingey, S. V., Tomb, J. F., Gordon, M. P., Olson, M. V., and Nester, E. W. (2001) The genome of the natural genetic engineer Agrobacterium tumefaciens C58, Science 294, 2317-2323.

[11] DeSanti, C. L., and Strohl, W. R. (2003) Characterization of the Streptomyces sp. strain C5 snp locus and development of snp-derived expression vectors, Appl. Environ. Microbiol. $69,1647-1654$. 